\title{
焊接电弧监测技术研究现状及展望”
}

\author{
蒋 凡 ${ }^{1,2}$ 李元锋 ${ }^{1}$ 陈树君 1 \\ (1. 北京工业大学汽车结构部件先进制造技术教育部工程研究中心 北京 100124 ; \\ 2. 哈尔滨工业大学先进焊接与连接国家重点实验室 哈尔滨 150001)
}

\begin{abstract}
摘要: 对焊接过程中的电弧信息进行实时监测和采集分析, 是保证弧焊质量、提升弧焊技术水平的重要途径。随着测量技术 和测量方法的不断发展, 对焊接电弧复杂信息进行分类提取的手段也逐步发展。文中从电弧热源的物理特性出发, 综述今年 来国内外开展的电弧焊接过程监视测量的研究现状, 主要包括以探针法和纹影法为代表的主动信息采集技术和以电弧电信 号、声信号和光信号采集为代表的被动信息采集技术，分类介绍典型焊接电弧监测技术的原理、特点和研究现状，并对该领 域的下一步发展趋势进行预测和展望。
\end{abstract}

关键词: 电弧焊; 焊接电弧监测; 电弧信息采集; 弧焊过程控制

中图分类号: TG444

\section{Current Situation and Prospects of Welding Arc Monitoring Technology}

\author{
JIANG Fan ${ }^{1,2}$ LI Yuanfeng ${ }^{1}$ CHEN Shujun ${ }^{1}$ \\ (1. Engineering Research Center of Advanced Manufacturing Technology for Automotive Components of Ministry \\ of Education, Beijing University of Technology, Beijing 100124; \\ 2. State Key Laboratory of Advanced Welding Production Technology, \\ Harbin Institute of Technology, Harbin 150001)
}

\begin{abstract}
The real-time information monitoring acquisition and analyze of arc in welding process, is a key way to ensure welding quality, and to enhance the level of arc welding technology. With the rapid development of measuring technique and measuring methods, the method to obtain valuable information, after classifying complicated arc information, has made great progress. This article starts from the physical properties of arc, summarizing the situation of arc working process monitoring and measurement at home and abroad. The arc monitoring and measurement mainly includes probe method and schlieren method, representative of active measurement, also the arc electrical signal, sound signal and light signal acquisition, representative of passive measurement. This article after recommending the theory, characteristics and research status of each welding arc measurement technology, the forecast of the development trend and prospects of this field are given in the end.
\end{abstract}

Key words: arc welding; welding arc monitoring; information acquisition of welding arc; arc welding process control

\section{0 前言}

自 1802 年瓦西里彼得罗夫发现了持续放电现 象并提出电弧在焊接加工中的应用潜力之后, 电 弧作为焊接热源在汽车、军事、航空航天和高端 装备制造 ${ }^{1}$ 等领域获得了广泛应用。传统自由电弧 虽然因其能量密度相对较低、能量分布不均匀等 弱点, 近年来受到激光、电子束等高能束热源的 冲击; 但对比高能束热源带来的对工作环境、加

* 国家自然科学基金(51375021)、北京市自然科学基金(3172004)和先进焊 接与连接国家重点实验室开放课题研究基金(AWJ-16-M06)资助项目。 20170523 收到初稿, 20170725 收到修改稿
工材料、装配精度等限制条件，以铇极惰性气体 保护焊(Tungsten inert gas welding, TIG)为代表的 传统电弧焊接工艺以技术成熟、操作简便、适应 性强、低投入高产出等优势, 继续在各个领域发 挥着不可替代的作用。

电弧焊接过程是一个复杂的物理变化过程, 焊 接电弧作为焊接过程能量传输的载体, 包含着大量 有关焊接过程稳定性、焊接质量的信息, 其工作状 态对于工件的焊接质量有着重要的影响。对焊接过 程中的电弧信息进行实时监测和采集分析, 是保证 弧焊质量、提升弧焊技术水平的重要途径。然而弧 焊过程中伴随着超高温度、大量电磁和辐射干扰及 电弧热物性耦合变化的复杂特性, 使其自身的反应 
过程复杂、受到的影响因素众多、难以对真实电弧 信息进行准确鉴别, 这些都使得对于电弧信息获取 难度大大增加。为了解决这些问题, 学者们针对焊 接电弧这一极端环境的监测技术开展深入研究, 根 据电弧信息的不同载体, 针对焊接电信号、焊接电 弧摄像观测、焊接电弧光信号及电弧物理性质的直 接测量等方面进行了不断的探索。根据电弧信息获 取的方式, 可将电弧监测技术划分为主动监测和被 动监测两大类, 文中从电弧热源的物理特性出发, 对典型焊接电弧监测技术的原理、特点及应用进行 了分类介绍, 并对该领域的未来发展趋势及值得关 注的问题进行了展望, 以期对焊接电弧测量监控及 电弧焊接技术的发展及其推广应用起到一定的促进 作用。

\section{1 电弧热源的物理特性}

焊接电弧作为电能向热能转换的载体, 电弧 的电、光、声等信息中包含着大量关于电弧状态 的信息 ${ }^{[1]}$, 如图 1 所示。焊接时, 电流击穿气体 介质形成电弧, 电弧作为回路的一部分将其自身 信息以电信号的形式表征出来; 弧柱中的中性粒 子在电场和电弧热效应的作用下被激发成一次电 离离子和二次电离离子, 据其当前状态辐射出不 同波长不同强度的弧光信号; 电弧等离子体高速 运动并冲击熔池, 电弧自身及带动熔池产生强烈 的振荡, 将其信息以声信号的形式传递出来。电 弧击穿气体环境时自身的尖端放电特性及其与周 围环境换热后达到局部热力学平衡时在电弧工作 区域内产生的大温度梯度, 使电弧中与温度直接 相关的热物理参数均形成明细梯度, 为电弧的主 动测量提供了便利条件。如何有效地利用焊接电 弧的特性实现对焊接电弧燃弧过程及状态的控 制, 一直是焊接工作者研究的目标。在电弧能量 变换中产生的这些物理现象是电弧信息传递的载 体, 也是电弧测量的首选对象。因此, 在对电弧 热源的物理特性进行研究的基础上, 焊接工作者 根据不同的应用目标, 针对电弧的宏观电信号、 图像和光学信息、声信号及物性梯度等进行了深 入的研究并开发了针对性的测量方法, 文中根据 电弧信息获取方式的不同将这些方法归纳为主动 监测和被动监测两大类, 并对其原理、特点和应 用进行了分类介绍, 并对焊接电弧监测技术的发 展趋势进行了评述。

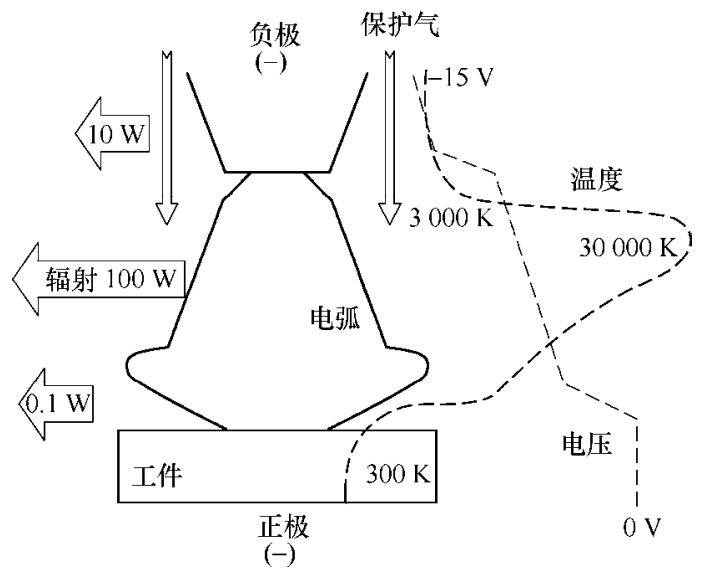

图 1 焊接电弧燃烧示意图

\section{2 电弧焊接过程的被动监测}

电弧的被动监测, 就是采集焊接过程中电弧向 外界传播的包含当前状态信息的伴生信号, 如电信 号、声信号、光谱信息、图像信息等, 从而获取监 测目标相关的特征信息。被动监测过程通常为非接 触监测且不会干扰电弧工况和测量结果, 因此具有 较高的准确性和工程适用性。但是电弧主动释放的 特性信号往往伴随着大量干扰和噪声，如何快速准 确地分辨出电弧特征信号并及时反馈给控制系统是 被动监测方法最为关注的内容。

\section{1 电信号监测}

在电弧焊接过程中, 电弧电压及焊接电流因其 易于采集和提取出与焊接过程相对应的特征信息, 被认为是弧焊质量监测和过程控制方面最为常用的 信息源。目前对电弧电流和电压参数的分析主要从 密度、动特性、周期变化等方面入手，通过特征点 的数理统计、曲线拟合、频谱分析、小波分析等提 取特性信号，在弧焊质量评定和焊接工艺改进方面 发挥了重要作用。

电弧电信号的时域波动是电弧动态变化的直接 表征, 根据电压、电流时域相图可以有效判断出电 弧焊接过程的稳定性。哈尔滨工业大学的白岩等 ${ }^{[2]}$ 根据熔化极等离子弧焊接的电压、电流时域相图, 对焊接过程的不稳定电弧电信号进行了分析。在熔 化极气体保护焊中, 通过对电信号的波动情况进行 统计分析, 可提取出电弧电流电压在动态变化过 程中的特性值, 对焊接工艺评定与改进起到指导 作用 ${ }^{[3]}$ 。在等离子弧穿孔焊接过程中，单纯的时域 分析或频域分析均难以捕捉这一非稳态过程的动态 变化。清华大学王海燕等 ${ }^{[4]}$ 将时域和频域结合, 如 图 2 所示, 截取一段时间内的电流信号做傅里叶变 换，从穿孔阶段的电信号频谱信息中提取的谱峰信 
号幅值和频率能有效检测熔池小孔的大小, 为实现 等离子穿孔焊接的熔透控制提供有效的反馈信息。 此外, 通过小波分析可以有效地在消除电信号噪声 的同时, 较好地保留信号的突变部分, 从而改善电 弧电信号中的特征信息的提取效果。华南理工大学 的易志平等 ${ }^{[5]}$ 用小波分析处理了 $\mathrm{CO}_{2}$ 焊的电流、电 压信号, 在不失真的情况下去除了高频噪声, 保持 了信号突变部分不失真, 改善了信号的质量, 使信 号特征更加明显。

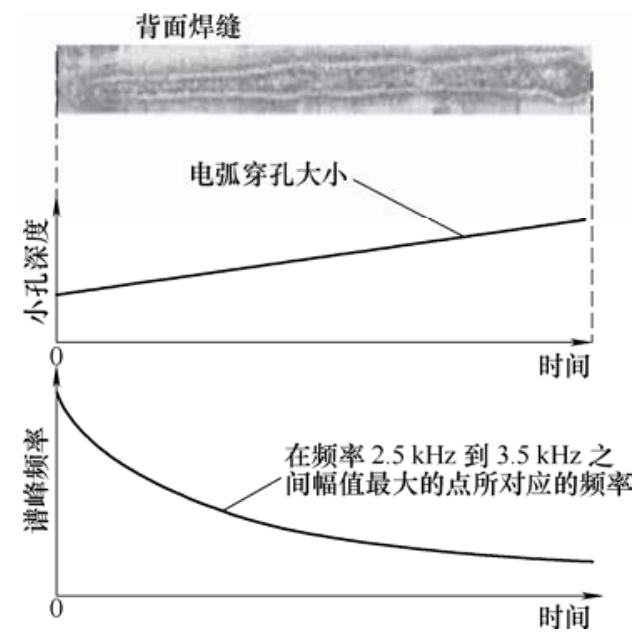

图 2 等离子弧穿孔特征信号

电弧电信号采集技术比较成熟, 采集系统响应 时间短, 分辨率高, 精度高, 并且可以有效避免强 电和弱电信号的干扰。由于电信号具有较高的时间 分辨率, 研究人员利用电信号的特征值来监控焊接 过程质量, 并且这种方法一直处于发展中。目前, 无论是在研究焊接电弧特性, 还是在弧焊实时反馈 控制中, 用电信号作为参考都是最常用、最便捷的 方法。但是在实际工业应用中, 焊接现场通常存在 大量干扰, 导致采集到的电压及电流信号品质下降, 增大了信号分析的难度。此外, 电信号检测需要依 赖离线软件以及丰富的专业知识辅助以得到正确的 认识, 使其应用范围受到限制。

\section{2 声信号监测}

电弧焊接过程中对熔池的持续冲击以及电弧 内部自身的高频振荡若发生在声波频率范围内, 则会将当前焊接过程的特征信息以声波的形式表 现出来 ${ }^{[6]}$, 如焊接动态参数变化, 电弧行为, 熔滴 过渡方式等, 是一种典型的非平稳随机信号, 其良 好的时间分辨率和不受弧光、烟尘和飞溅等影响的 特性使其成为焊接过程中具有实际应用价值的潜在 源信号, 随着现代音频采集技术和信号处理降噪技 术的快速发展, 以电弧声信号为信息源的焊接过程 监测技术开始受到研究人员的关注。
在等离子弧穿孔焊接过程中, 熔池的振动是 声音信号低频分量的主要来源, 清华大学的王耀 文等 ${ }^{[7]}$ 据此通过离散加窗傅里叶变换, 在低频信号 中捕捉到反应穿孔过程的信息, 图 3 所示的低频分 量 $\mathrm{A}$ 在不同穿孔阶段中有较明显变化, 为利用声信 号监测等离子弧穿孔提供了可靠的参照量。在铇级 氩弧焊焊接过程中, 电弧内部的等离子流是声源激 励源。上海交通大学的吕娜等 ${ }^{[8]}$ 以脉冲非熔化极焊 接气体保护焊为研究对象, 通过电弧声与功率的对 比, 发现电弧声信号的声源激励来源与电弧能量的 变化有明显相关性, 并且随着电弧从未熔透到熔透 状态, 声压逐渐增大, 据此提取特征后实现熔透状 态识别。对于 $\mathrm{CO}_{2}$ 气体保护焊, 试验证明电弧声主 要源于燃弧与短路过程 ${ }^{[9]}$, 并将电弧声信号与焊接 工艺参数、熔滴过渡形式建立了对应关系 ${ }^{[10]}$ 。兰州 理工大学的马跃洲 ${ }^{[11]}$ 分析了 $\mathrm{CO}_{2}$ 短路过渡的电弧声 信号, 由于电弧声的频谱特征与焊接状态高度相关, 将频带能量做小波包多层分解后得到飞浌特征信号, 一定程度上实现了对焊接飞溅的识别。

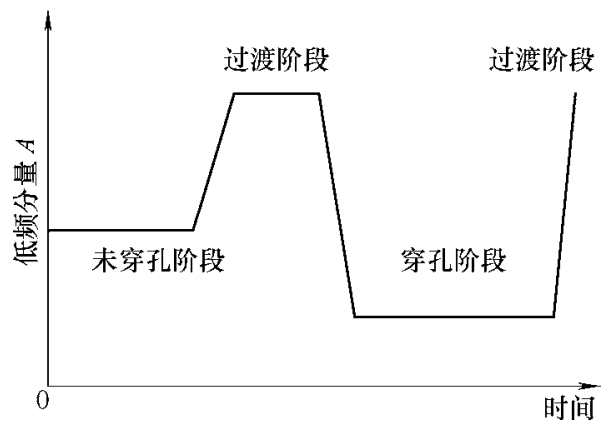

图 3 A 算法随穿孔过程的变化

但是在实际生产应用中, 无法避免的机器噪声 和人为的干扰使声音传感检测出来的声音信号发生 扰动, 导致电弧声信号的准确采集相对困难, 且声 信号具有高度的复杂性和非线性, 难以直观、准确 地将其变化规律与焊接过程的动态变化相联系, 且 电弧声信号的发生机制和传播特性等问题尚待进一 步研究, 使得难以直接利用电弧声信号进行焊接过 程监控。

\section{3 电弧视觉传感}

与电弧电信号、声信号相比, 良好的空间分辨 率使电弧视觉信息具有独特的优势, 此外视觉图像 传感器不与焊接回路接触, 在信息传感过程中免于 受到多种干扰因素特别是复杂的工作环境和随机信 号的影响, 因此被研究者广泛使用于电弧形貌监测 和熔透状态监测。在现代高速摄像技术的支持下， 动态图像采样的频率和精度得到大幅提升, 丰富了 电弧视觉传感的时间、空间信息。近年来帮助焊接 
研究者采集到大量关于电弧形态、熔滴过渡、电弧 极性变化、电弧穿孔过程的瞬态信息 ${ }^{[12]}$ 。电弧视觉 图像具有直观和信息量丰富的特点, 使利用视觉图 像传感技术监测电弧形态, 研究电弧特性已经成为 焊接电弧监测领域的一个重要方法。但是, 目前电 弧视觉传感技术难以定量测定电弧发光区域与边 界, 随着曝光时间的增加, 电弧发光区域面积变大, 同样当光圈等拍摄条件变化时, 发光区域面积也会 发生改变。北京工业大学的郭珍珍 ${ }^{[13]}$ 采集了图 4 所 示的电弧图像, 并提出一种通过图像融合算法, 将 不同曝光时间的图像融合后得到图 5 所示的清晰焊 接过程图像, 在保留电弧真实信息的基础上提高了 电弧视觉图像的动态范围 ${ }^{[13]}$ 。
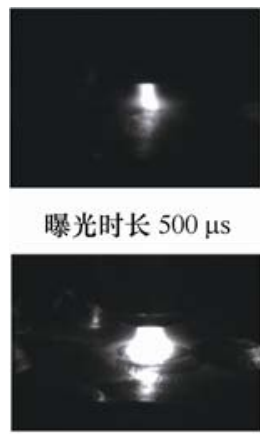

曝光时长 $650 \mu \mathrm{s}$

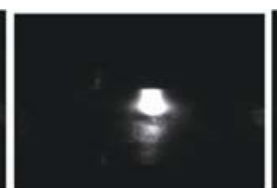

曝光时长 $550 \mu \mathrm{s}$

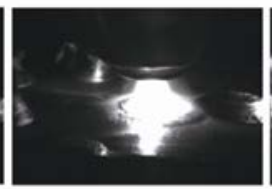

曝光时长 $750 \mu \mathrm{s}$

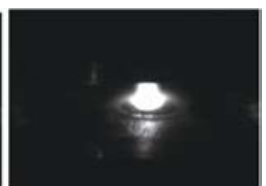

懪光时长 $600 \mu \mathrm{s}$

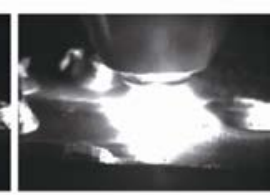

懪光时长 $800 \mu \mathrm{s}$
图 4 不同曝光时间下的电弧图像

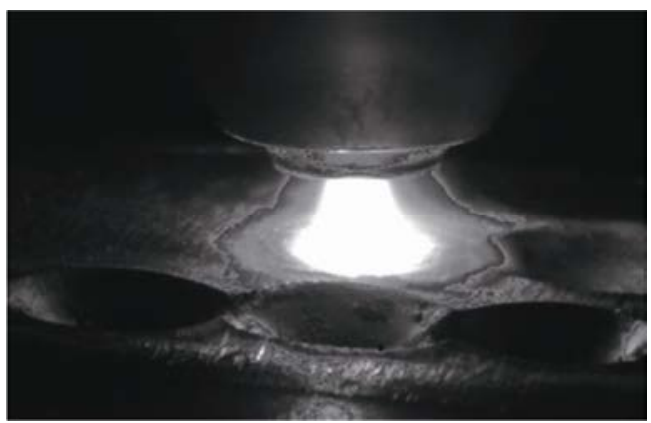

图 5 电弧融合效果图

\section{4 光谱诊断}

电弧放电发出强烈的光辐射, 在发射光谱中具 有很强的连续谱和特征谱, 长期以来被研究者用于 电弧等离子体的物理的定性和定量分析, 电弧的光 谱诊断法是利用光谱仪直接收集图 6 所示的电弧等 离子体发出的光谱辐射。电弧内部剧烈的热运动产 生了能量较低的连续谱, 在自身的物理性质和环境 状态的影响下, 电弧发射谱线有一定的宽度与轮廓, 图中谱线强度是表示光谱线能量的物理量, 半高宽 是指在谱带峰值高度一半时的峰宽, 结合相关光谱 理论后, 可以得到反映电弧温度、电流密度的定量 信息。

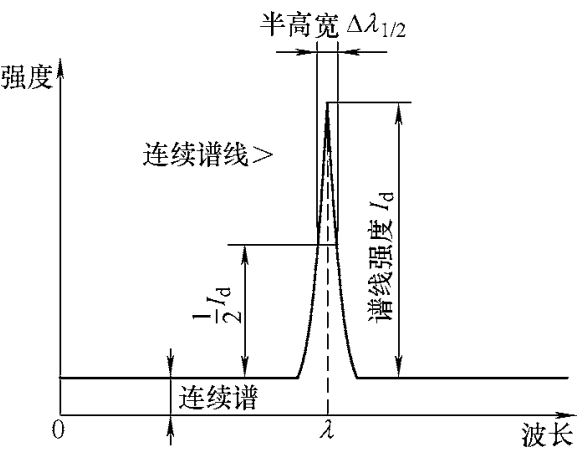

图 6 电弧光谱结构示意图

光谱仪是将复合光分成一系列波长的谱线及其 强度的一种光学仪器。在光谱仪的测量过程中, 由 于电弧内部成分、温度的极不均匀性, 需要对电弧 各个空间位置进行扫描。按照扫描方式, 光谱仪测 量方法主要分为成像扫描法、转镜扫描法、狭缝扫 描法。澳大利亚的 HAIDAR ${ }^{[14]}$ 设计了一种成像扫描 法测量系统, 通过透镜将电弧成像在一个平面上, 如图 7 所示, 步进电动机带动电弧在空间上移动, 光谱仪负责采集电弧各空间位置的光谱分布。随着 技术发展, 研究人员利用光纤将电弧光送入光谱仪, 这一改动大幅提高了系统的灵活性, 目前移动光纤 探头就可完成对电弧的扫描。此方法具有较高的精 度, 但逐点扫描造成时间分辨率较差, 测量结果不 能保证具有一致性 ${ }^{[15]}$ 。宋永伦 ${ }^{[16]}$ 设计了一种图 8 所 示的转镜扫描法装置, 通过旋转反射镜将不同位置 的电弧光传入光谱仪, 由于镜子转动速度有限, 难 以提高时间分辨率，导致测量结果依旧存在一定误 差。为了克服以上缺点, CELVAREZ 等 ${ }^{[17]}$ 搭建了一 种狭缝扫描法测量系统, 如图 9 所示, 在狭缝作用 下, 一个截面上的电弧光送入光谱仪, 在光栅的作 用下，原本一个截面下一维的电弧光被展开成坐标 轴为空间和波长的光谱图像, 通过移动狭缝完成电 弧整体的扫描 ${ }^{[15]}$ 。这种方法一次曝光可以采集一个 截面的电弧光谱信息, 极大提高了时间分辨率, 但 是不同位置的电弧光进入光谱仪时会相互干扰, 影 响精度。

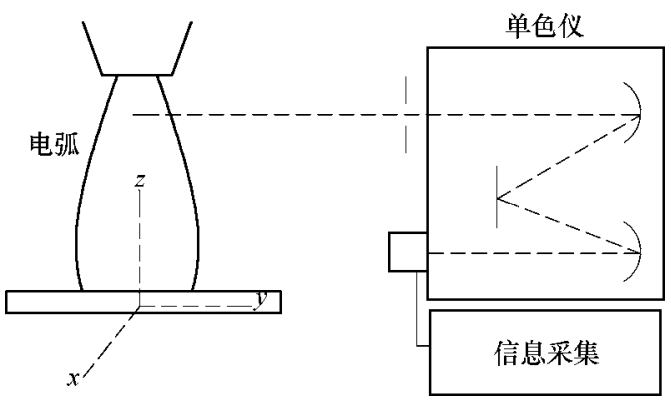

图 7 成像扫描法装置示意图 


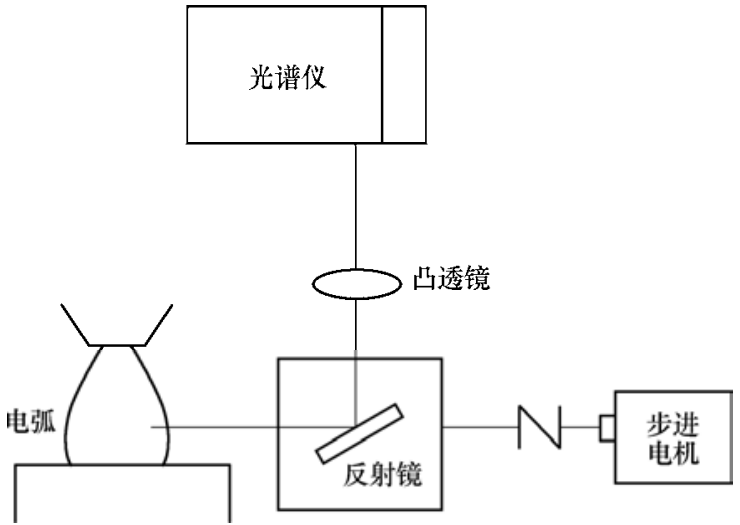

图 8 转镜法装置示意图

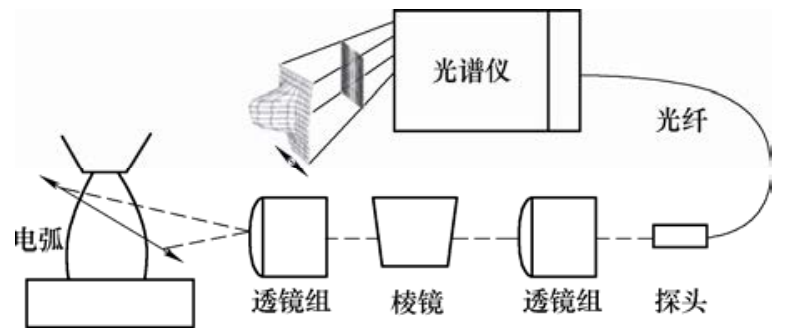

图 9 狭缝扫描法装置示意图

电弧内部的局部热力学平衡状态决定了电弧光 谱强度与电弧等离子体内部温度、粒子浓度和成分 等存在密切关系 ${ }^{[18]}$ 。在焊接过程中, 电弧温度场和 电流密度场是影响焊接质量的重要因素, 对电弧热 源的了解有助于我们进一步改进焊接工艺, 因此在 光谱诊断中, 测量电弧密度场和温度场是电弧光谱 诊断的重点, 其中史塔克展宽法用来求解电弧电子 密度, 谱线绝对强度法、谱线相对强度法是测量电弧 温度的主要方法, 史塔克展宽法利用谱线在电子作 用下产生的史塔克展宽来计算电子密度 ${ }^{[19]}$, 这种方 法要求光谱仪具有较高精度, 并且所选谱线具有适 当的强度和良好的轮廓。谱线绝对强度法是采集单 条谱线的绝对强度值来计算电弧温度 ${ }^{[20]}$, 这种测温 原理虽然相对简单, 但是发射系数的测量误差以及 粒子跃迁概率的不可靠性造成结果误差较大 ${ }^{[15]}$, 所 以一直以来绝对强度法在电弧测量领域的应用较 少。相比之下, 谱线的相对强度法应用较为常用 ${ }^{[21]}$, 如图 10 所示, 通过同时采集同一空间位置的两条或 多条谱线信息, 提高了后期理论计算的准确度 ${ }^{[22]}$ 。 日本大阪大学的 HIRAOKA 等应用谱线相对强度法 和多种测温方式对纯 $\mathrm{Ar}$ 气氛的 TIG 电弧和 $\mathrm{Ar}-\mathrm{H}_{2}$ 混合气体 TIG 电弧进行测量, 用相对强度法在两种 电弧距铇级下方 $1 \mathrm{~mm}$ 处的径向温度测量结果如图 11 所示, 通过不同测温方法结果的对比, 证明了相 对强度法的准确性 ${ }^{[23]}$ 。上海交通大学的斯红等 ${ }^{[22]}$ 分析了相对强度法的谱线选取原则, 从 ArII 特征谱 线中选取 9 条合适谱线, 然后对 TIG 电弧温度场进
行测量, 得到如图 12 所示的温度场信息 ${ }^{[21]}$, 并通 过与前人试验结果的对比, 最终证明了所选谱线的 可靠性。

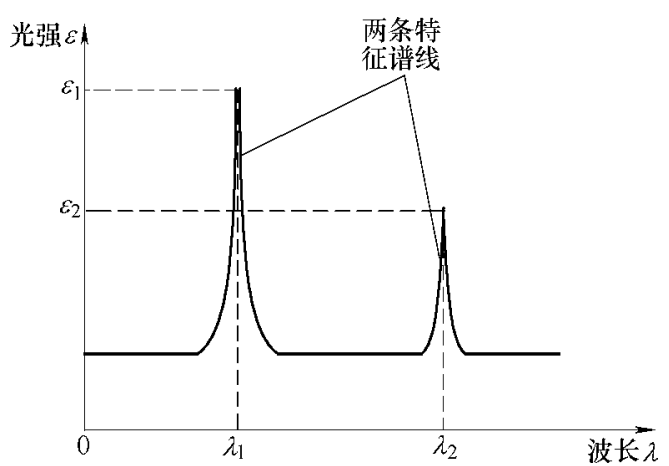

图 10 谱线相对强度法
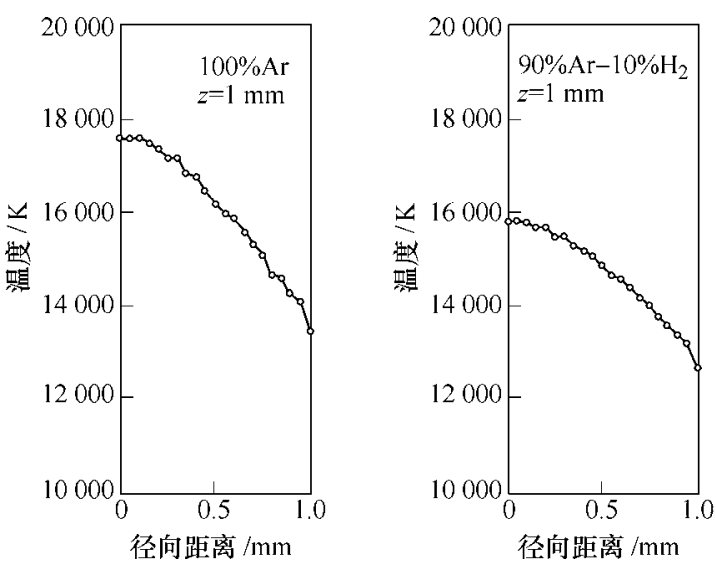

图 11 TIG 电弧 $1 \mathrm{~mm}$ 处径向温度分布

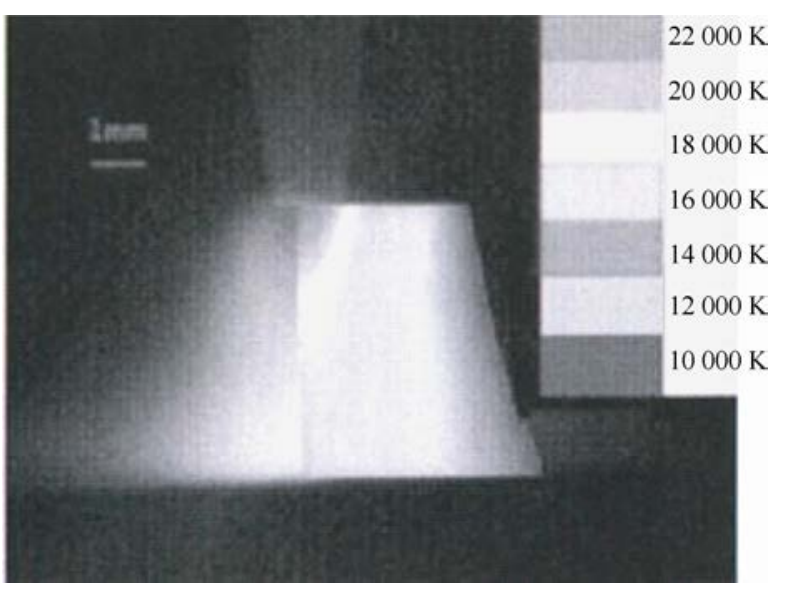

图 12 TIG 电弧温度场信息

电弧内部上万度的高温导致常规方法难以采集 到其内部信息, 光谱诊断因其无干扰, 灵敏度和分 辨率高的优点, 目前已成为电弧等离子体诊断的主 要方法。但是这种方法也具有一定的局限性, 虽然 现代光谱测量设备大幅缩短了波段的扫描时间, 但 是在电弧诊断过程中依旧需要耗费大量时间才能完 成对电弧整体的扫描, 最终造成这种方法的时间分 辨率较差。另一方面, 光谱诊断受限于等离子体物 理的理解和计算方法的准确性, 目前还没有完善的 
等离子体热力学计算方法, 跃迁概率等不确定性较 高的物理参数给计算结果带来较大误差。

\section{5 窄带光谱图像技术}

将先进的视觉图像采集技术和光谱学理论相结 合, 窄带光谱图像技术利用特定波段内的光谱强度 信息完成对电弧特性的监测, 在一次曝光中收集电 弧不同位置处的光强信息, 解决了光谱法空间分辨 率低的问题。测量系统如图 13 所示, 窄带滤光片使 部分波段的光吸收或反射, 将另一部分光送入 CCD 相机中 ${ }^{[15]}$ 。

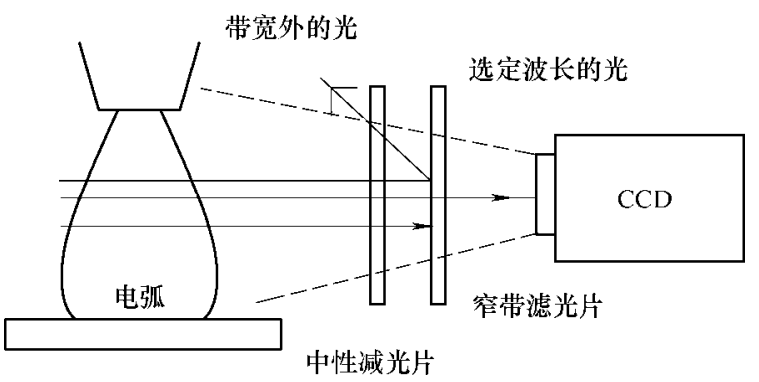

图 13 窄带光谱图像采集

在监测系统中加入现代分光装置后, 窄带光谱 图像技术还可以同时采集多条谱线的电弧光谱信 息, 利用分光镜或刀锋棱镜等光学元件, 使电弧光 等能量分成两束, 然后分别被两台 CCD 相机接收。 大阪大学的 TANAKA 课题组搭建了图 14 所示的分 光系统, 分光镜使两台 CCD 相机同时测量熔化极惰 性气体保护焊熔滴过渡过程中电弧的 $\mathrm{Ar}$ 线谱和 $\mathrm{Fe}$ 谱线的发射系数场, 得到图 15 所示的电弧整体温度 和 Fe 浓度的动态可视化信息 ${ }^{[24]}$ 。

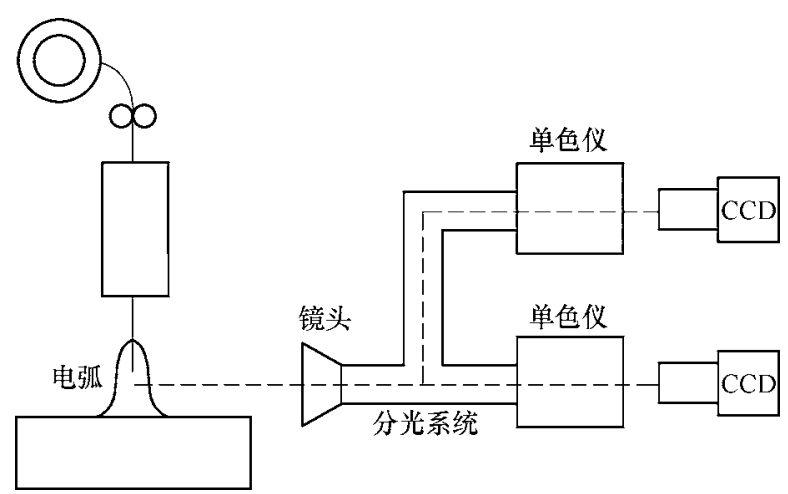

图 14 多谱线同步采集

在窄带滤光片选取特征谱线后, CCD 相机采集 的窄带图像可以看作为宏观的单一谱线强度分布。 在谱线自吸收效应较弱时, 如图 16 所示, CCD 感 光元件一个像素点采集的光强 $O P$ 可以近似为直线 $A B$ 上电弧发射系数的积分 ${ }^{[25]}$, 求逆还原截面上各 个坐标点的发射系数, 最后根据等离子体的发射系 数与温度的对应关系来求解电弧的温度分布。窄带
光谱图像技术在计算发射系数与温度的对应关系 时, 通过归一化处理, 减少了跃迁概率等不确定度 较高的参数对光谱理论计算造成的误差。

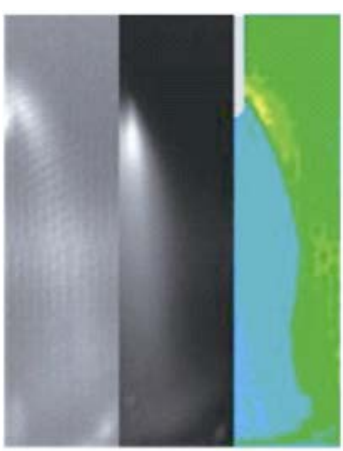

(a) $0 \mathrm{~ms}$

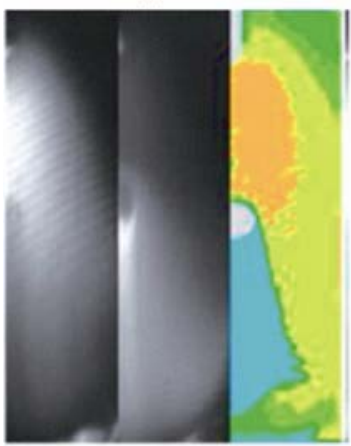

(c) $2 \mathrm{~ms}$

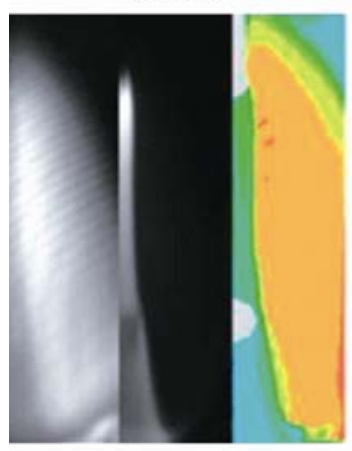

(e) $4 \mathrm{~ms}$

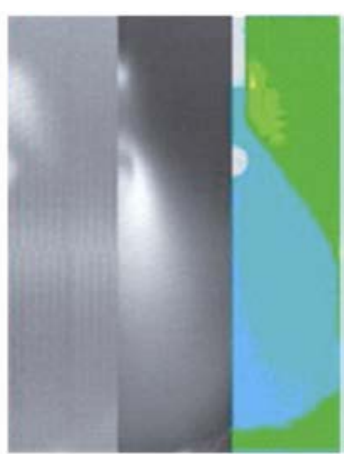

(b) $1 \mathrm{~ms}$

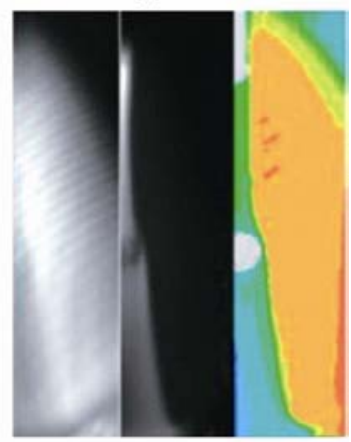

(d) $3 \mathrm{~ms}$
图 $15 \mathrm{Ar}$ 谱线(左图)、 $\mathrm{Fe}$ 谱线(中图)的光强分布和 电弧的整体温度分布(右图)

半径

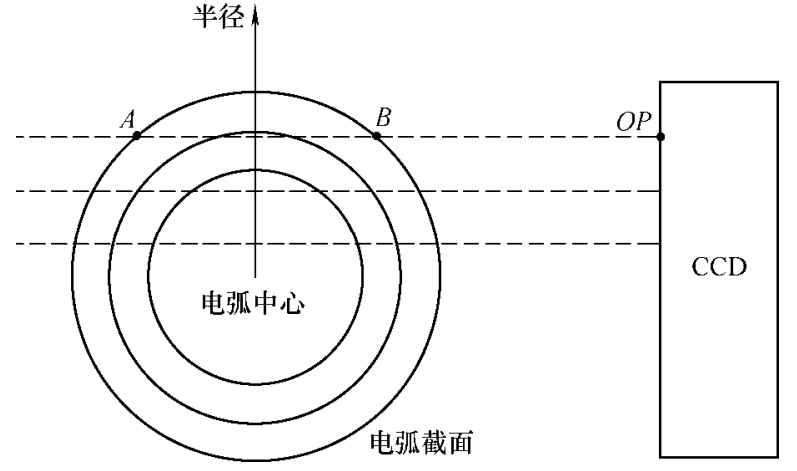

图 $16 \mathrm{CCD}$ 相机采集发射系数

窄带光谱图像技术因单一谱线的图像使信息更 加集中, 克服了图像方法仅能作为电弧形态定性观 测的缺陷, 满足了电弧放电机构及其尺度的定量化 
的认识需求, 在保证一定准确性的基础上, 有着较 广的适用性, 它良好的时间、空间分辨率使其可以 较好反映脉冲、变极性焊接电弧的物理变化过程, 一定程度上弥补了传统电信号、声信号和光谱信息 的不足, 有着广阔的应用前景。

但是在实际测量中, 电弧内部粒子必须满足局 部热力学平衡状态, 同时发射系数和温度的对应关 系不是单调的 ${ }^{[26]}$, 当电弧温度超过标准温度时发射 系数下降, 导致发射系数最大点偏离轴心, 此外, 传统的窄带光谱图像技术在测量传统的静态电弧 时, 可以假设电弧热呈环形分布, 因此只需要采集 一个角度的电弧光强信息, 就可以通过逆积分运算 还原电弧发射系数场, 但是不规则的非对称电弧截 面, 需要多角度的光强信息来进行截面还原。研究 人员致力于解除窄带光谱图像技术中轴对称条件的 制约, 为了还原非对称电弧发射系数场, 哈尔滨工 业大学的熊俊 ${ }^{[27]}$ 发明了一种电弧旋转装置, 通过旋 转电弧, 来使 CCD 相机在不同方位角下完成电弧的 谱线信息采集, 试验系统如图 17 所示, 最后根据不 同角度的电弧窄带图像还原电弧截面发射系数。此 外, 大阪大学的 HIRATA 课题组设计了多台 CCD 相 机联动的电弧发射系数场测量系统, 如图 18 所示, CCD 相机以电弧为中心等间隔排布, 同时在不同角 度下采集电弧窄带图像 ${ }^{[28]}$, 以移动的 TIG 电弧作 为监测对象, 试验得到如图 19 所示的电弧温度分 布 ${ }^{[29]}$, 这种电弧监测装置在保证良好的时间、空间 分辨率的基础上还原电弧的三维全貌。

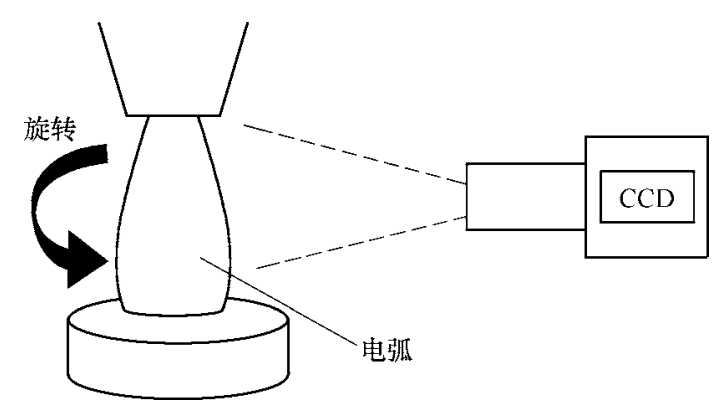

图 17 多角度旋转测量电弧

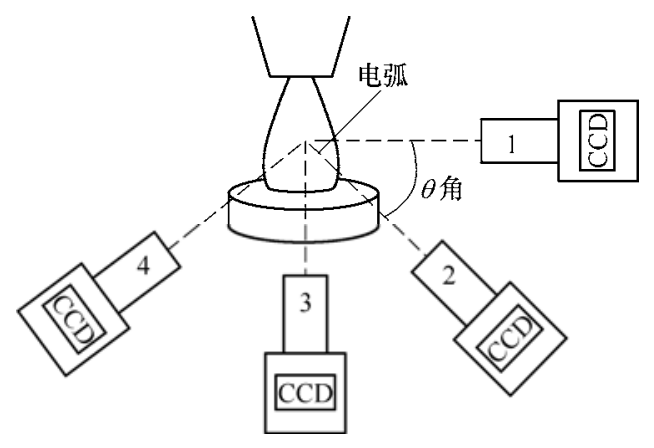

图 18 多台 CCD 同步测量电弧

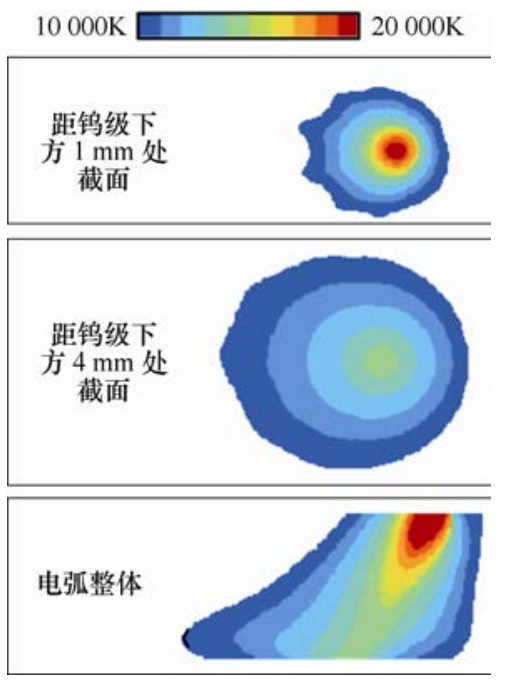

图 19 TIG 电弧温度分布

\section{3 电弧焊接过程的主动监测}

电弧的主动监测是将主动信号投射入电弧等离 子体, 接收反馈信号并分析电弧对信号的影响从而 得出当前电弧的物理特性。主动监测方法具有响应 迅速、后期数据处理简单等优势, 但在监测过程中 可能会对电弧产生干扰, 并且结果受环境等因素影 响较大。

\section{1 探针法}

探针法就是将静电探针送入电弧等离子内, 通 过探针测量的电信号, 可以确定出等离子体空间电 位、电子温度、电子密度和离子密度等参数 ${ }^{[29]}$ 。良 好的三维空间分辨率使其不用还原电弧截面信息, 但是探针法作为一种接触式监测方法势必会改变探 针周围的电弧热物理参数。

传统的静电探针结构简单，一般只有探针头端 部可以导电, 如图 20 所示, 在深入电弧后, 根据探 针的 V-I 特性曲线计算等离子体物理参数。

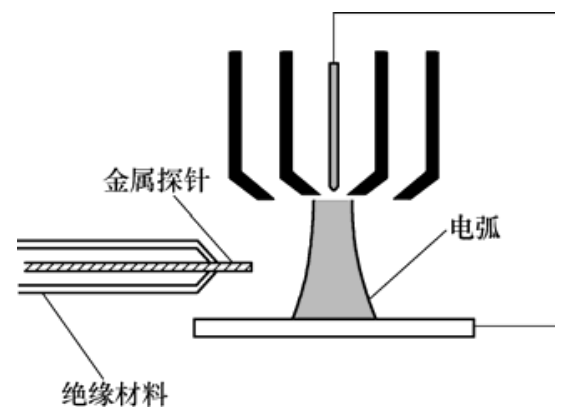

图 20 金属探针结构

但是传统的静电探针在测量电弧等离子体时, 电弧的高温特性导致探针寿命问题难以解决。为了 保护探针不被烧损，英国的 FANARA 等 ${ }^{[30]}$ 开发了 一种扫动式探针, 如图 21 所示, 探针高速横向旋转 
进入电弧, 减小探针与高温等离子体的接触时间, 但是探针在旋转过程中夹杂的外部气体进入电弧内 部, 对电弧产生了更大干扰。对此, 兰州理工大学 发明了一种新型低扰动探针如图 22 所示 ${ }^{[31]}$, 用铝 丝作为探针, 在两侧牵引轮带动下进入电弧。用铝 丝表面阳极氧化镀膜处理代替了传统绝缘管, 在满 足绝缘性能的基础上大幅度减小了绝缘物尺寸, 延 探针中轴方向的运动方式减少了扫动式探针带入电 弧的外部气体, 在探针不被烧损的情况下有效降低 了探针对电弧的干扰。

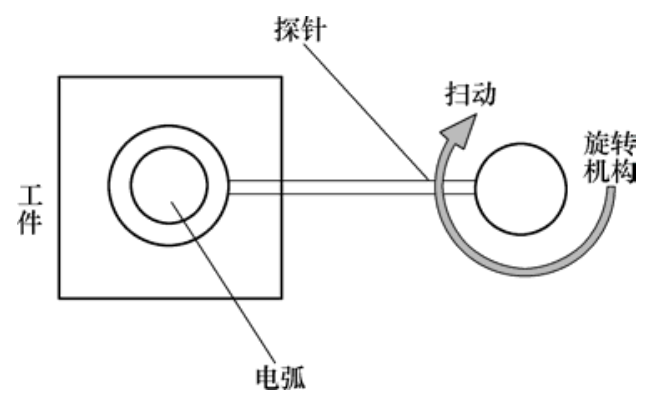

图 21 扫动式探针结构示意图

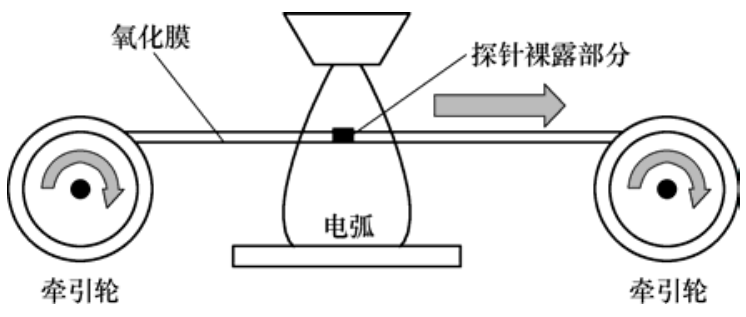

图 22 一种低扰动探针的结构示意图

在静电探针不连接外部电源时, 插入电弧等离 子体后依旧会产生的伏特级鞘层电压 ${ }^{[32]}$ 。肯塔基大 学的 ZHANG 等 ${ }^{[33]}$ 用静电探针监测等离子弧焊的穿 孔过程, 如图 23 的静电探针被放置在等离子云中, 等离子云等价电阻值在穿孔的过程中出现规律性变 化, 根据检测电路中的电阻压降就可完成对等离子 弧的穿孔状态的监测。

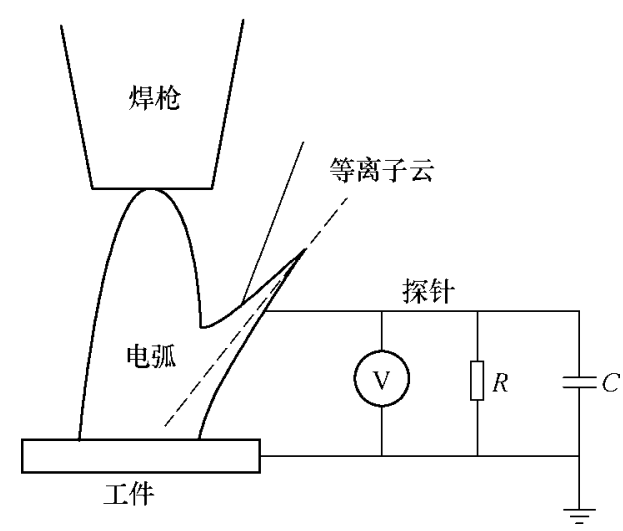

图 23 等离子云团测量系统示意图

\section{2 纹影监测}

纹影法利用流场折射率正比于气流密度的基本 原理对电弧进行测量, 将电弧流场中密度梯度的变 化转变为记录平面上相对光强的变化 ${ }^{[34]}$ 。纹影系统 如图 24 所示, 刀口平行于成像屏移动, 以至于挡住 大部分直射光, 保留部分衍射光。已有研究者应用 纹影仪观测等离子弧焊的电弧流动、激光焊的保护 气体流动 ${ }^{[35-36]}$ 。德国的 SIEWERT 等 ${ }^{\left[{ }^{[37]}\right.}$ 用纹影仪监 测 MIG 焊接过程中的气体流动, 在不同试验系统参 数下拍摄纹影图像, 并说明了纹影仪的相关设置参 数对纹影图像的影响, 试验中采集的纹影图像如图 25 和图 26 所示 ${ }^{[38]}$ 。

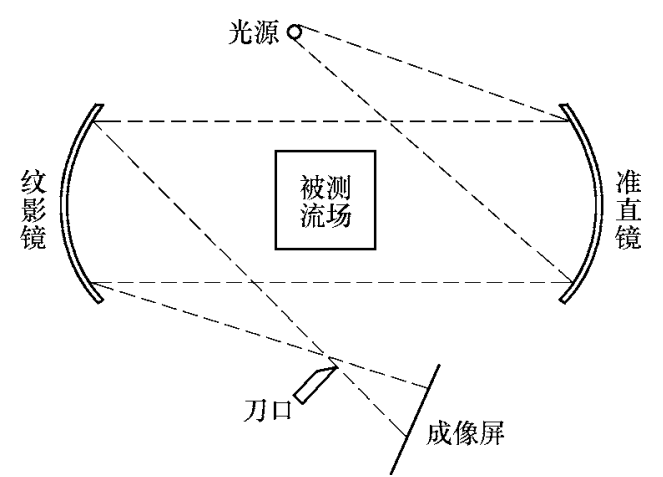

图 24 纹影法测量系统示意图

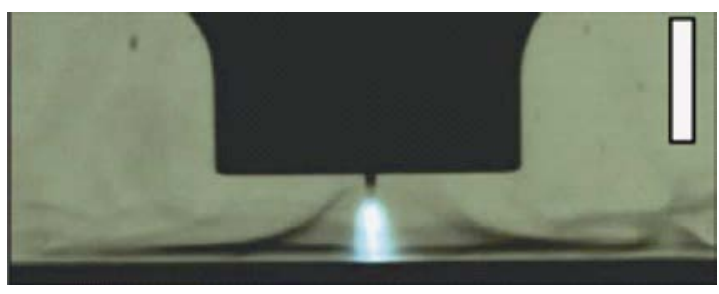

图 25 坚刀口所采集的 MIG 焊纹影图像

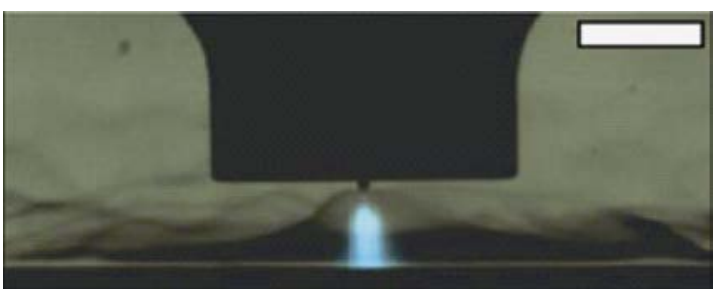

图 26 横刀口所采集的 MIG 焊纹影图像

干涉纹影法也是纹影法中在电弧测量中应用较 广泛的一种方法, 用激光光源照射电弧等离子体介 质, 在介质中电子的作用下, 光源通过介质时产生 偏折并在干板上形成干涉图像。干涉图像中包含了 电弧各个空间位置的成分信息, 最后通过干板上的 干涉条纹和等离子体折射率与温度的对应关系还原 电弧的温度分布 ${ }^{[39]}$ 。干涉法跟光谱法一样要求被测 等离子体处于局部热力学平衡状态 ${ }^{[38]}$, 并且干涉法 灵敏度较高, 拥有较好的时间空间一致性, 一次测 量能获得全场信息, 对待测对象不产生干扰, 但光 
学设备需要具有较高的稳定性。哈尔滨工业大学的 姚庆泰 ${ }^{[40]}$ 利用干涉纹影法对常规 TIG 电弧和超声 -TIG 电弧的温度进行了测量, 设计了图 27 所示的 一种脉冲激光二次曝光试验系统, 两束光经过相同 的光程照射在全息干板上发生干涉, 电弧各个空间 位置的光波信息被记录到干板上, 并用火焰温度场 对系统的准确性进行验证, 证明这种二次曝光全息 干涉法适用于电弧等离子体的温度诊断。

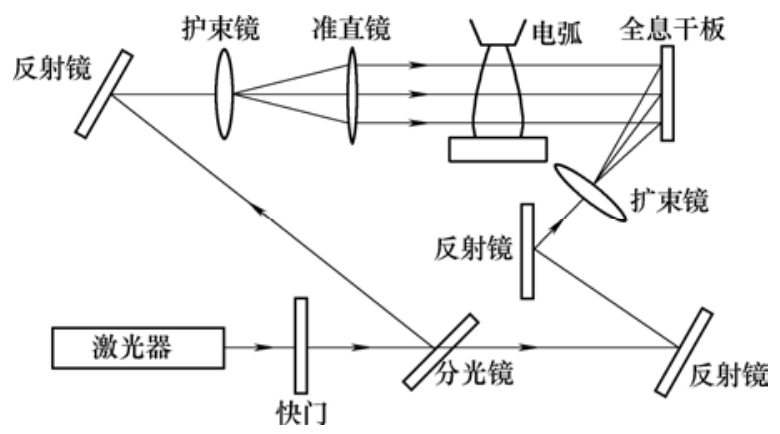

图 27 脉冲激光二次曝光系统示意图

\section{4 焊接电弧监测技术的发展趋势}

为了提升焊接工艺水平, 目前出现了越来越多 的新型焊接形式, 如多电极复合焊接 ${ }^{[41]}$ 、真空铇级 氩弧焊 ${ }^{[42]}$ 等。这些新型工艺的出现给弧焊接过程监 测提出了更高的要求。

(1) 复杂电弧形态还原。动态电弧、新型复合 电弧等非轴对称电弧加大了电弧监测的难度。若以 电弧为旋转中心, 用光谱仪逐角度扫描非对称电弧 会花费大量的时间, 电弧的不稳定性会使测量结果 带有很大误差。另外, 目前具有非对称截面还原能 力的 iradon 变换、ART 算法等重建法需要庞大的后 期计算，不适用于焊接的实时监控。

(2) 丰富的时间、空间信息。动态电弧的内部 参数场处于不断变化中, 因此从时间和空间中获取 有效的信息是保证测量准确性的必然要求, 给目前 技术带来了新的挑战。首先, 良好的时间分辨率是 电弧动态测量的基本要求。电信号、声信号和视觉 图像由于具有良好的时间分别率，可以完成对电弧 宏观状态或形态的监测。与之相比, 光谱仪的时间 分辨率较差, 难以完成对动态电弧的整体监测。其 次, 电弧内部大温度梯度、成分梯度的物理特性对 监控技术提出了空间分辨的需求, 极大地推动了窄 带光谱技术的发展。

(3) 监测结果的准确性。传统电弧信息的采集 分析可通过多种手段进行比对验证, 还可采集有限 信息作为数学模型的边界条件从而得出目标数据并 对测量结果的可靠性进行验证 ${ }^{[43]}$ 。但是多电极电弧
等创新电弧热源打破了传统的电弧燃弧理论, 创新 型的电弧结构也导致监测手段宽乏、三维重建和数 学建模更加困难等问题。

\section{5 结论}

随着各类传感技术、信息提取技术、计算机运 算能力的发展和更新, 电弧监测技术进一步升级, 在焊接过程质量控制、电弧特性研究等领域中起到 越来越重要的作用。为了使电弧监测技术在焊接领 域实现更广泛的应用，需要不断提高从焊接过程中 采集的时间、空间信息量来完善对电弧动态过程的 监测。在焊接电弧宏观动态监测不能满足要求的情 况下, 以电弧光谱为信息载体, 视觉图像为记录媒 介的窄带光谱图像技术给我们带来了更全面的动态 信息，有着广阔的发展前景。

\section{参 考 文 献}

[1] 肖天骄. 焊接 TIG 电弧的导电机构及其能量分布的研 究[D]. 北京: 北京工业大学, 2013.

XIAO Tianjiao. A study on conductive mechanism of TIG welding arc and its energy distribution[D]. Beijing: Beijing University of Technology, 2013.

[2] 白岩, 高洪明, 路浩, 等. 基于 LabVIEW 的熔化极等 离子弧焊接电弧电信号分析 [J]. 焊接学报, 2006, 27(8): 59-62.

BAI Yan, GAO Hongming, LU Hao, et al. Analysis of Plasma-MIG arc signal based on LabVIEW[J]. Transactions of The China Welding Institution, 2006, 27(8): 59-62.

[3] 李桓, 陈埒涛, 李国华. 不同电感条件下焊接电弧电信 息的统计分析 [J]. 焊接技术，2004，33(6)：7-9.

LI Huan, CHEN Lietao, LI Guohua. Statistical analysis of welding arc electrical information under the conditions of different induction[J]. Welding Technology, 2004, 33(6): 7-9.

[4] 王海燕, 陈强, 孙振国, 等. 等离子焊接熔池小孔尺寸 的电弧信号检测 [J]. 焊接学报, 2000, 21(3): 24-26.

WANG Haiyan, CHEN Qiang, SUN Zhenguo, et al. Detection of keyhole size by arc signal processing[J] Transactions of The China Welding Institution, 2000, 21(3): 24-26.

[5] 易志平, 薛家祥. $\mathrm{CO}_{2}$ 焊电信号的小波分析 [J]. 华南理 工大学学报, 2002, 30(6): 27-29.

YI Zhiping, XUE Jiaxiang. Wavelet analysis of electric signals in $\mathrm{CO}_{2}$ welding[J]. Journal of South China University of Technology, 2002, 30(6): 27-29.

[6] 兰虎. 基于电弧声信号特征 MIG 焊熔透状态模式识别 
[D]. 哈尔滨: 哈尔滨理工大学, 2009.

LAN Hu. Penetration state recognition of mig welding based on signal characteristics of arc sound[D]. Harbin: Harbin University of Science and Technology, 2009.

[7] 王耀文, 陈强, 孙振国, 等. 等离子弧焊接穿孔行为 的 声信号传感[J]. 机械工程学报, 2001, 37(1): 53-56. WANG Yaowen, CHEN Qiang, SUN Zhenguo, et al. Sound sensing of the keyhole behaviors in plasma arc welding[J]. Chinese Journal of Mechanical Engineering, 2001, 37(1): 53-56.

[8] 吕娜. 基于电弧声信号的铝合金脉冲 GTAW 熔透特征 识别及其实时控制研究[D]. 上海: 上海交通大学, 2014. LÜ Na. Recognition and real-time control of penetration characteristics of al alloy pulsed GTAW process based on the arc audio information[D]. Shanghai: Shanghai Jiao Tong University, 2014.

[9] GRAD L, GRUM J, POLAJNAR I, et al. Feasibility study of acoustic signals for on-line monitoring in short circuit gas metal arc welding[J]. International Journal of Machine Tools \& Manufacture, 2004, 44(5): 555-561.

[10] 温建力. MIG 焊电弧声实验初步探讨 [J]. 煤矿机械, 2009(9): 99-100.

WEN Jianli. MIG welding arc sound experimental preliminary study[J]. Coal Mine Machinery, 2009(9): 99-100.

[11] 马跃洲. 基于电弧声信号的 CO 焊质量监控方法研究 [D]. 兰州: 兰州理工大学, 2005.

MA Yuezhou. Research on the methods for quality monitoring based on arc sound signal in $\mathrm{CO}_{2}$ arc welding[D]. Lanzhou : Lanzhou University of Technology, 2005.

[12] ZHANG Y M, WU L, CHEN D H, et al. Determining joint penetration in GTAW with vision sensing of weld face geometry[J]. Welding Journal, 1993, 72(10): S463-S469.

[13] 郭珍珍. 宽动态焊接过程监控系统研制[D]. 北京: 北京 工业大学, 2016.

GUO Zhenzhen. A multiple exposure images fusion used in welding control system[D]. Beijing: Beijing University of Technology, 2016.

[14] HAIDAR J. Large effect of cathode shape on plasma temperature in high-current free-burning arcs[J]. Journal of Physics D Applied Physics, 1994, 27(3): 555.

[15] 马税良. 基于图像采集的 TIG 焊接电弧动态光谱诊断 研究[D]. 哈尔滨: 哈尔滨工业大学, 2009.

MA Shuiliang. Time resolved spectroscopic diagnosis of TIG welding arcs by monochromatic imaging[D]. Harbin: Harbin Institute of Technology, 2009.

[16] 宋永伦. 焊接电弧等离子体的光谱诊断法及其应用的 研究[D]. 天津: 天津大学, 1990 .
SONG Yonglun. Spectroscopic study and its application in welding arc plasma[D]. Tianjin: Tianjin University, 1990.

[17] ÁLVAREZ R，RODERO A， QUINTERO M C. An Abel inversion method for radially resolved measurements in the axial injection torch[J]. Spectrochimica Acta Part B Atomic Spectroscopy, 2002, 57(11): 1665-1680.

[18] HADDAD G N, FARMER A J D. Temperature determination in a free-burning arc. I. Experimental techniques and results in argon[J]. Public Choice, 1984, 62(2): 139-151.

[19］张旺, 华学明, 潘成刚, 等. 基于 Stark 展宽的 TIG 焊 接电弧三维电子密度测量研究 $[\mathrm{J}]$. 光谱学与光谱分析, 2012, 32(10): 2601-2604.

ZHANG Wang, HUA Xueming, PAN Chenggang. The reconstruction of welding arc 3D electron density distribution based on stark broadening[J]. Spectroscopy and Spectral Analysis, 2012, 32(10): 2601-2604.

[20] CHIANG $\mathrm{W} \mathrm{T}$, GRIEM H R. Observations of line-continuum interference effect in the wings of argon ion lines[J]. Journal of Physics B Atomic \& Molecular Physics, 1978, 11(24): L761-L764.

[21] 斯红. 基于标准温度法 TIG 电弧温度场诊断技术研究 [D]. 上海：上海交通大学，2013.

SI Hong. Temperature field of TIG arc based on FOWLER-MILNE method[D]. Shanghai: Shanghai Jiao Tong University, 2013.

[22] 斯红, 华学明, 张旺, 等. 基于 Boltzmann 光谱法的焊 接电弧温度场测量计算 $[\mathrm{J}]$. 光谱学与光谱分析, 2012, 32(9): 2311-2313.

SI Hong, HUA Xueming, ZHANG Wang, et al. Welding arc temperature field measurements based on Boltzmann spectrometry[J]. Spectroscopy and Spectral Analysis 2012, 32(9): 2311-2313.

[23] SHIWAKU T. Determining temperature distributions of gas tungsten arc (TIG) plasma by spectroscopic methods[J]. Welding International, 1997, 11(9): 688-696.

[24] TSUJIMURA Y, NAKATANI M, TANAKA M. Analysis of metal vapor behavior in gas shielded arc welding with consumable electrode by imagining spectroscopy[J]. Tetsu-to-Hagane, 2012, 98(10): 534-540.

[25] 殷咸青, 孙江涛, 张建勋. 纵向磁场作用下的铇极氩弧 焊电弧温度场 $[J]$. 西安交通大学学报, 2013，47(3): 85-89.

YIN Xianqing, SUN Jiangtao, ZHANG Jianxun. Arc temperature distribution of gas tungsten arc welding in external longitudinal magnetic field[J]. Journal of Xi'an Jiaotong University, 2013， 47(3): 85-89.

[26] 马税良, 高洪明, 张广军, 等. 阿贝尔逆变换数据处理 算法在电弧诊断中的应用 $[J]$. 光学学报, 2007, 27(9): 
1633-1638.

MA Shuiliang, GAO Hongming, ZHANG Guangjun, et al. A data processing method for Abel inversion in arc plasma diagnostics[J]. Acta Optica Sinca，2007，27(9): 1633-1638.

[27] 熊俊. 基于 ART 算法的双铇极耦合电弧温度场光谱诊 断[D]. 哈尔滨: 哈尔滨工业大学, 2010.

XIONG Jun. Spectroscopic diagnosis of temperature distribution of twin-electrode TIG coupling arc based on art algorithm[D]. Harbin: Harbin Institute of Technology, 2010.

[28] NOMURA K, KISHI T, SHIRAI K, et al. Temperature measurement of asymmetrical pulsed TIG arc plasma by multidirectional monochromatic imaging method[J]. Welding in the World Le Soudage Dans Le Monde, 2014, 59(2): 283-293.

[29] 陆云松. 低温等离子体朗缪探针诊断电路研究[D]. 沈 阳: 东北大学, 2008.

LU Yuansong. Study of the Langmuir probe diagnostic circuit for the low-temperature plasma[D]. Shenyang: Northeastern University, 2008.

[30] FANARA C, VILARINHO L. Electrical characterization of atmospheric pressure arc plasmas[J]. The European Physical Journal D, 2004, 28(2): 241-251.

[31] 李渊博. 绝缘片约束 TIG 电弧的静电探针分析及其在 超窄间隙中的加热特性[D]. 兰州: 兰州理工大学, 2013.

LI Yuanbo. The analysis of constricted TIG arc by insulating plate with electrostatic probe and arc heating characteristic in ultra-narrow gap[D]. Lanzhou: Lanzhou University of Technology, 2013.

[32] 易小林. 等离子弧焊中等离子云检测机理与模糊控制 的研究[D]. 天津: 天津大学, 2002.

YI Xiaolin. Theory of the detection of plasma cloud and fuzzy control in plasma arc welding[D]. Tianjin: Tianjin University, 2002.

[33] ZHANG Y M, ZHANG S B, LIU Y C. A Plasma cloud charge sensor for pulse keyhole process control. Measurement Science and Technology, 2001, 12: 1-6.

[34] 林杏全, 陈武秀. 纹影法及其应用[J]. 华中师范大学学 报，1980(1): 45-51.

LIN Xingquan, CHEN Wuxiu. The schlieren method and application[J]. Journal of Central China Normal University, 1980(1): 45-51.

[35] 黄瑞, 胡治华, 陈恺. 激光焊接保护气体流态的纹影法 研究[J]. 焊接技术，2007(2): 8-11，79.

HUANG Rui, HU Zhihua, CHEN Kai. The study of laser welding shielded gas by schlieren[J]. Welding Technology, 2007(2): 8-11, 79.

[36] MCCLURE J C, HOU H R. A study of gas flow pattern, undercutting and torch modification in variable polarity plasma arc welding[J]. Journal of Environmental Management, 1994, 164: 67-73.

[37] SIEWERT E, WILHELM G, HÄSSLER $M$, et al. Visualization of gas flows in welding arcs by the schlieren measuring technique: The influence of typical welding parameters on the gas flow for the GTAW, GMAW, and PAW processes is demonstrated using the high-speed schlieren technique[J]. Welding Journal, 2014(Suppl.): $1-5$.

[38] 薛海涛, 李桓, 李俊岳, 等. 电弧等离子体折射率的理 论计算[J]. 机械工程学报, 2004, 40(8): 49-53.

XUE Haitao, LI Huan, LI Junyue, et al. Theoretic calculation of arc plasma refractive index[J]. Chinese Journal of Mechanical Engineering, 2004, 40(8): 49-53.

[39] 李俊岳, 李益山. 电弧等离子体的单波长激光平晶干涉 诊断[J]. 计量学报, 1984(2): 124-130.

LI Junyue, LI Yishan. Diagnostics of the electric arc plasma by mono-wavelength laser interferometry with optical flat[J]. Acta Metrologica Sinica, 1984(2): 124-130.

[40] 姚庆泰. 超声辅助焊接电弧等离子体全息干涉研究[D]. 哈尔滨：哈尔滨工业大学, 2016.

YAO Qingtai. Research on holographic interference of ultrasonic assisted welding arc plasma[D]. Harbin: Harbin Institute of Technology, 2016.

[41] 陈姬, 宗然, 武传松, 等. TIG-MIG 复合焊电弧间相互 作用对焊接过程的影响 [J]. 机械工程学报, 2016, 52(6): 59-64.

CHEN Ji, ZONG Ran, WU Chuansong, et al. Influence of arcs interaction on TIG-MIG hybrid welding process[J]. Journal of Mechanical Engineering, 2016，52(6): 59-64.

[42] 陈树君, 王建新, 蒋凡, 等. 空心铇极中心负压电弧基 础特性研究[J]. 机械工程学报, 2016, 52(2): 7-12. CHEN Shujun, WANG Jianxin, JIANG Fan, et al. Research of hollow tungsten central negative pressure arc welding characteristic[J]. Journal of Mechanical Engineering, 2016, 52(2): 7-12.

[43] 丁雪萍, 李桓. 焊接电流影响 GMAW 双丝焊电弧等离 子体的数值模拟研究 [J]. 机械工程学报, 2016, 52(16): 71-76.

DING Xueping, LI Huan. Numerical analysis for effect of welding current on arc plasma in double-wire GMAW[J]. Journal of Mechanical Engineering, 2016, 52(16): 71-76.

作者简介: 蒋凡, 男, 1987 年出生, 博士, 讲师。主要研究方向为焊接 电弧物理、创新电弧热源、创新焊接技术和先进焊接方法与应用。

E-mail: jiangfan@bjut.edu.cn

陈树君(通信作者), 男, 1971 年出生, 博士, 教授。主要研究方向为现 代焊接设备及自动化, 电力电子技术与电磁兼容, 先进焊接工艺。发表 论文 100 余篇。

E-mail: sjchen@bjut.edu.cn 